\title{
SAW Sensor for Mercury Vapour Detection
}

\author{
K. JaseK* ${ }^{*}$ S. Neffe And M. PASternak \\ Military University of Technology, S. Kaliskiego 2, 00-908 Warsaw, Poland
}

\begin{abstract}
Mercury is the element commonly applied in industry. Because of poisoning properties the monitoring of the mercury vapour concentration in the environment is very important. Mercury forms amalgams in connection with gold that causes a change in the electrical resistance and mass of the gold specimens. This effect was applied to measure mercury vapour concentration in the environment using surface acoustic wave technology. Two kinds of surface acoustic wave mercury sensors have been described in the paper. First one utilizes a thin film of gold deposited between aluminium interdigital transducers and reflectors of two-port surface acoustic wave resonator, and the second one golden interdigital transducers and reflectors without any sensitive film between them.
\end{abstract}

PACS: 43.35.Pt, 46.35.+z, 68.35.Ja

\section{Introduction}

Although it is well known that mercury and its compounds can cause serious poisoning, the element is still used in many branches of industry. Natural level of $\mathrm{Hg}$ in air averages between 1 and $2 \mathrm{ng} / \mathrm{m}^{3}$. Measuring such low levels requires preliminary mercury vapour concentration and sophisticated analytical equipment. Current laboratory techniques for determining the presence of the element are mainly based on spectroscopic and electrochemical methods $[1,2]$. While these techniques are very sensitive, they are not suitable for in situ monitoring. Therefore the research project discussed in the paper aimed at producing small and cheap mercury sensors, that can be used directly in an area where mercury vapour monitoring is necessary (e.g. work places). In Poland, the occupational exposure limits for $8 \mathrm{~h}$ and for $15 \mathrm{~min}$ are $0.025 \mathrm{mg} / \mathrm{m}^{3}(3 \mathrm{ppb})$ and $0.2 \mathrm{mg} / \mathrm{m}^{3}(24 \mathrm{ppb})$ respectively [3]. The monitoring of mercury vapour concentration at those limits is possible with simple sensors and in-line preconcentrators.

The most commonly used $\mathrm{Hg}$ sensors are based on quartz crystal microbalance (QCM) devices $[4,5]$. These sensors contain a layer of sorbent for elemental mercury vapours, which changes its physical and/or chemical properties in the sorption process. Therefore, this layer does not only play the role of a receptor, but it is also a preconcentrator. As an absorbent both metals, such as $\mathrm{Ag}, \mathrm{Au}, \mathrm{Pd}, \mathrm{Pt}$, and other elements and compounds, such as $\mathrm{C}, \mathrm{PdCl}_{2}, \mathrm{Pd}$ /polypyrole [5-9] are often used. However, the most commonly applied material for the detection of mercury vapour is pure gold. The mechanism of sorption of mercury vapour in a golden layer was studied, among others, in $[5,10-13]$. It was postulated that

\footnotetext{
* corresponding author; e-mail: kjasek@wat.edu.pl
}

the adsorbed $\mathrm{Hg}$ atoms are mobile and move across the surface until they encounter a discontinuity, such as a surface defect or grain boundary, which makes possible their diffusion into the crystal by place exchange mechanism. A monolayer of $\mathrm{Hg}$ and subsequent, loosely bound layers (depending on the concentration of mercury vapour) are formed on the surface of the gold. Assuming that the mercury atoms are stacked side by side on the surface of a flat gold film with no surface morphology, the monolayer of $\mathrm{Hg}$ is estimated to be equal to $285 \mathrm{ng} / \mathrm{cm}^{2}$ [5]. For a low concentration of mercury vapour the diffusion reaches only 5-6 atomic layers of the gold [11].

The desorption process proceeds in reverse order whereby the removal of $\mathrm{Hg}$ atoms above the first monolayer takes place according to first order kinetics with the activation energy equal to the heat of absorption $\Delta H \approx 15 \mathrm{~kJ} / \mathrm{mol}$ [5]. The second stage of the desorption process is characterized by secondary kinetics with activation energy increasing as the coverage of the gold by $\mathrm{Hg}$ atoms decreases. At that stage, in the centres of the absorption, the $\mathrm{Hg}$ atoms move from the bulk of gold to the surface, then move along the surface and eventually are desorbed. Quantitative desorption of $\mathrm{Hg}$ from a layer of gold occurs at temperatures above $200^{\circ} \mathrm{C}$ [12]

Previous work on acoustic elemental mercury vapour sensors was focused on QCM sensors, with little emphasis on surface acoustic wave (SAW) sensors, even though they are often much more sensitive when similar sorptive layers are used. Only a few papers can be found on the SAW mercury vapour sensors $[13,14]$ and all of them utilized SAW delay lines. This paper presents two mercury vapour sensors based on two-port SAW resonators with aluminium and golden electrodes. First one utilizes a thin film of gold deposited between aluminium interdigital transducers (IDTs), and the second one golden IDTs only. 


\section{Experimental}

Two kinds of sensors - one with a layer of gold deposited between aluminium IDTs and the other with gold IDTs and reflectors without a sorptive layer were investigated. As sensors bases, the SAW synchronous multimode two-port resonators on ST-cut quartz were used.

The first kind of structure has aluminium IDTs and operates at a centre frequency of about $196.8 \mathrm{MHz}$ with $12 \mathrm{~dB}$ insertion loss and $50 \Omega$ loaded quality factor equal to 12000 . The second one utilises golden IDTs and operates at a centre frequency of about $194.3 \mathrm{MHz}$, characterises $14 \mathrm{~dB}$ insertion loss and $50 \Omega$ loaded quality factor of about 11500 . The resonators have the same geometry. The difference between IDTs materials gives as a result the mirror symmetry of their frequency characteristics [15]. Both resonators were designed for gas sensors applications and have about $6 \mathrm{~mm}^{2}$ surface area between IDTs suitable for chemosensitive films depositing.

Measurements were performed for three different devices. In the first one $(\mathrm{Al}+\mathrm{Au})$ the golden $50 \mathrm{~nm}$ film having $4 \mathrm{~mm}^{2}$ area between aluminium IDTs was deposited using thermal evaporation. The atomic force microscopy (AFM) measurements revealed that the film had a $40 \%$ larger surface area than its projected value. The other two devices had no additional sorptive layers. One of them had golden $(\mathrm{Au})$, and the second aluminum $(\mathrm{Al})$ electrodes of IDTs. The last one was used as a reference device insensitive to mercury vapour. Each of resonators operates with three modes corresponding with the centre of the reflection structure band as well as with its low and high frequency edges. In all devices the higher frequencies were utilised because of the lowest insertion loss.

The sensors frequencies shifts were measured using a set of equal and independent SAW positive feed-back loop oscillators. The thermostated set gives the possibility to carry out simultaneous comparative measurements for different sensors pieces. In Fig. 1 the diagram of an individual oscillator is shown [16].

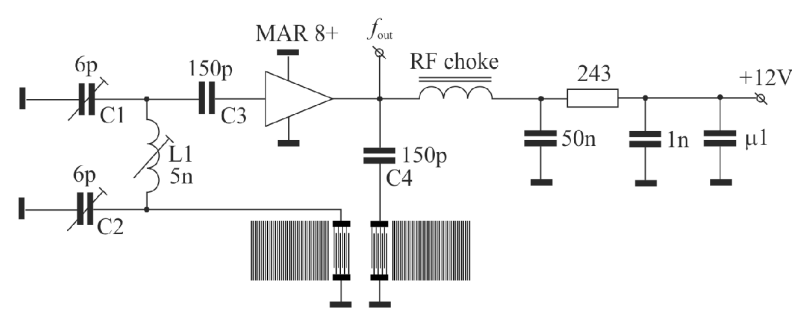

Fig. 1. SAW oscillator used in measurements.

Tuneable capacitors $\mathrm{C} 1, \mathrm{C} 2$ and inductor L1 are working as a phase shifter to fulfil the phase condition of the feed-back loop self-oscillation. The amplitude condition is provided here by an appropriate amplifier bias set. The values of block capacitors $\mathrm{C} 3, \mathrm{C} 4$ are chosen to suit the frequency range of the oscillator. Such a configuration is flexible enough to be used for both kinds of resonators described here.

The measurement setup consists of a thermostatic chamber housing SAW devices, a thermostatic diffusion source of mercury vapour and the flow controls system of $1 \%$ accuracy class. It allows to generate a desired mercury concentration in the carrier gas (Fig. 2) with a temperature stability of $\pm 0.3{ }^{\circ} \mathrm{C}$. Frequency shifts measurements were performed using HAMEG programmable counter HM8123 with $400 \mathrm{MHz}$ time base and $0.5 \mathrm{ppm}$ stability.

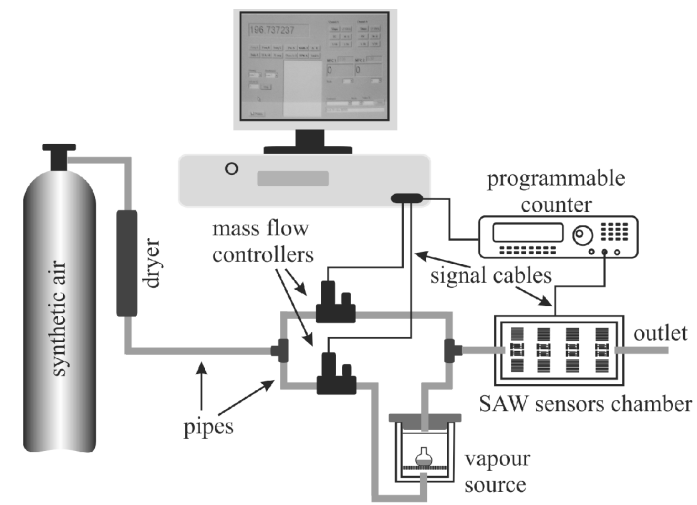

Fig. 2. The measurement setup.

The sensors response to the step-concentration of mercury vapour change was investigated at the beginning. Exemplary results are shown in Fig. 3.

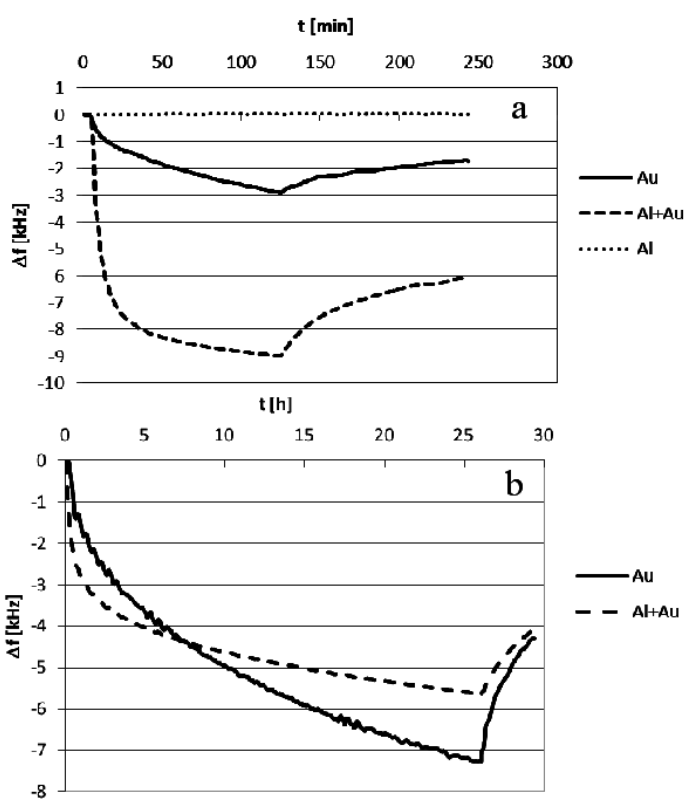

Fig. 3. The sensor response to the unit increase of mercury vapour levels at $60^{\circ} \mathrm{C}$ : (a) two-hour measurement, (b) 24-hour measurement.

After exposing the sensors to mercury vapour, rapid changes of the output signal for $\mathrm{Al}+\mathrm{Au}$ sensor were ob- 
served, although for the sensor with golden electrodes the changes were considerably slower. However, the $\mathrm{Al}+\mathrm{Au}$ sensor quickly enters the area of signal saturation and, after several hours of exposure, the Au sensor signal change was significantly greater than that for the sensor $\mathrm{Al}+\mathrm{Au}$. This is due to the fact that the initial period of exposure is dominated by the adsorption of $\mathrm{Hg}$ on the surface of gold and the surface area of the deposited layer is much larger than the surface area of the golden electrodes of resonator (both IDTs and reflectors). The massive electrodes are able to absorb more $\mathrm{Hg}$ than the deposited film of gold, while the time of diffusion of mercury atoms inside the electrodes is much longer than for surface adsorption. Figure $3 \mathrm{~b}$ shows that it takes at least several days to establish the thermodynamic equilibrium at temperature of the environment.

As the sensor should provide information on the presence of mercury within tens of seconds, the initial parts of the concentration curves, which correspond to the adsorption of $\mathrm{Hg}$ on the surface layer of gold, are of particular importance. Subsequently, the slow diffusion of $\mathrm{Hg}$ inside the gold electrodes can be advantageous for the construction of dosimeters for determining of the summary $\mathrm{Hg}$ dose absorbed by the sensor.

In view of the overlap of two parallel processes of $\mathrm{Hg}$ vapour sorption, each of which has a different activation energy, so the characteristics of such kind of sensors response are nonlinear (Fig. 4). The initial steep section of the curve corresponds to the first monolayer adsorption of $\mathrm{Hg}$, which is very strongly associated with the surface of the gold. The raising concentration of $\mathrm{Hg}$ vapour no longer causes a strong signal change because of the fact that other less-connected layers are subsequently forming. Against the background of the rapid signal change, resulting from the surface adsorption process, a slow decrease in the signal due to the diffusion processes is observed. The sensitivity of new sensors (not previously in contact with mercury vapour) in the initial period of exposure can be estimated to be around $20 \mathrm{~Hz} / \mathrm{ppb}$ for the $\mathrm{Al}+\mathrm{Au}$ sensor and $5 \mathrm{~Hz} / \mathrm{ppb}$ for $\mathrm{Au}$.

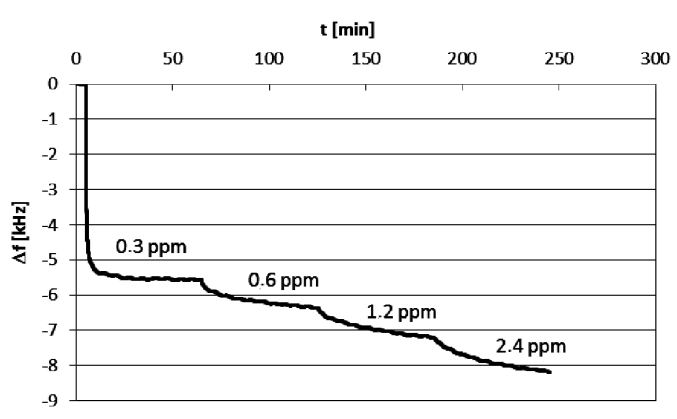

Fig. 4. Concentration characteristic of $\mathrm{Al}+\mathrm{Au}$ sensor.

After each series of measurements the sensors were regenerated at $60{ }^{\circ} \mathrm{C}$ under nitrogen atmosphere for several hours during which a partial desorption of $\mathrm{Hg}$ atoms from a layer of gold occurs. However, after each regeneration

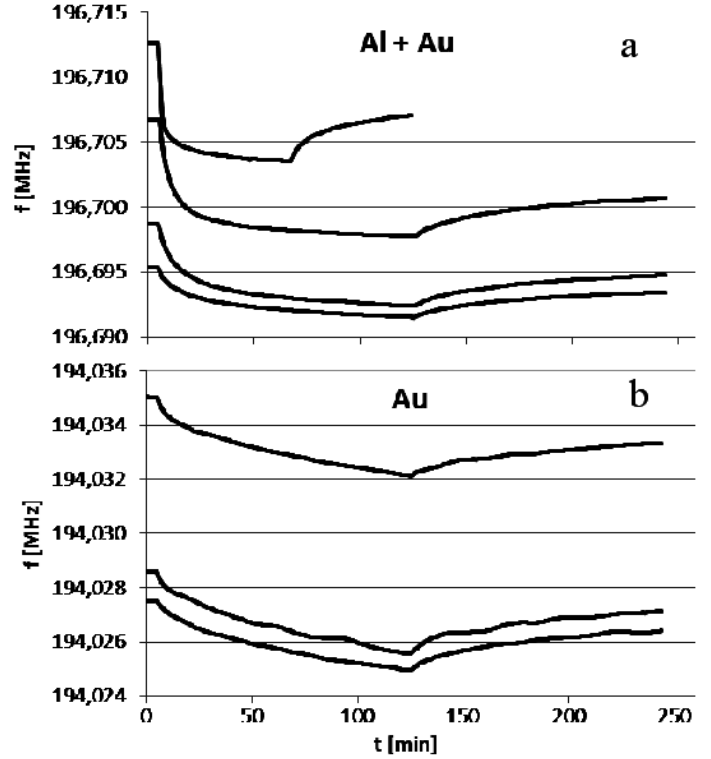

Fig. 5. The reduction in the sensor signal during subsequent exposures to mercury vapour.

process the signal attained even lower level (Fig. 5).

Subsequent measurements also gave smaller signal changes when the sensor was exposed to the same mercury vapour concentration.

\section{Conclusions}

In this study, two types of SAW mercury sensors were investigated. One was based on the two-port SAW resonator with golden film between aluminium IDTs and reflectors, while the second one was a resonator with golden IDTs and reflectors without any additional sorptive layer.

It was found that the first sensor is much more responsive to the presence of mercury vapour in the environment, but with long exposures of several hours. The signal changes were greater for the second sensor. In the initial period of exposure the sensitivity of the sensor with the golden film is about $20 \mathrm{~Hz} / \mathrm{ppb}$, while a sensor with golden electrodes only has a sensitivity of $5 \mathrm{~Hz} / \mathrm{ppb}$. Changes in temperature in the range of $20-60{ }^{\circ} \mathrm{C}$ do not have a significant influence on the performance of the sensors, which results from the fact that the change in signal is mainly due to the surface adsorption, which is much less sensitive to temperature than the bulk absorption.

There are two main disadvantages of the investigated sensors. Firstly, it is impossible to regenerate them at room temperature, secondly, the degree of the sensor saturation influences its concentration related characteristics. Therefore, such sensor can be used mainly for indicating the mercury vapour presence in the environment as well as for determining the absorbed dose of mercury, which depends not only on concentration but also on the exposure time. 


\section{References}

[1] W.L. Clevenger, B.W. Smith, J.D. Winefordner, Crit. Rev. Anal. Chem. 27, 3 (1997).

[2] T. Pustelny, A. Opilski, B. Pustelny, Acta Phys. Pol. A 114, A183 (2008).

[3] Act of Polish Ministry of Labour and Social Policy on occupational exposure limits (Journal of Laws of 2002, No. 217, item 1883).

[4] D.P. Ruys, J.F. Andrade, O.M. Guimarães, Anal. Chim. Acta 404, 95 (2000).

[5] Y.M. Sabri, S.J. Ippolito, J. Tardio, A.J. Atanacio, D.K. Sood, S.K. Bhargava, Sensors Actuators B 137, 246 (2009).

[6] P.D. Selid, H. Xu, E.M. Collins, M. Striped Face-Collins, J.X. Zhao, Sensors 9, 5446 (2009).

[7] E.J. Granite, H.W. Pennline, R.A. Hargis, Ind. Eng. Chem. Res. 39, 1020 (2000).

[8] Y.M. Sabri, R. Kojima, S.J. Ippolito, W. Wlodarski, K. Kalantar-Zadeh, R.B. Kaner, S.K. Bhargava, Sensors Actuators B: Chem. 160, 616 (2011).
[9] V.M. Mirsky, M. Vasjari, Comprehensive Anal. Chem. 49, 235 (2007).

[10] T. Morris, J. Sun, G. Szulczewski, Anal. Chim. Acta 496, 279 (2003).

[11] C. Battistoni, E. Bemporad, A. Galdikas, S. Kaciulis, G. Mattogno, S. Mickevicius, V. Olevano, Appl. Surf. Sci. 103, 107 (1996).

[12] T. Morris, J. Sun, G. Szulczewski, Anal. Chim. Acta 496, 279 (2003).

[13] J.J. Caron, R.B. Haskell, P. Benoit, J.F. Vetelino, IEEE Trans. Ultrason. Ferroelectr. Freq. Control 45, 361 (1998).

[14] B. Pustelny, T. Pustelny, Acta Phys. Pol. A 116, 383 (2009).

[15] W. Soluch, IEEE Trans. Ultrason. Ferroelectr. Freq Control 55, 696 (2008).

[16] K. Jasek, M. Pasternak, Europ. Phys. J. 154, 97 (2008). 